\title{
SAFETY-ORIENTED SPEED GUIDANCE OF URBAN EXPRESSWAY UNDER MODEL PREDICTIVE CONTROL
}

\author{
Chen, D. S.; Yu, X. X.; Hu, K. Q.; Sun, X. \& Xia, Y. Y. \\ School of Transportation Engineering, Huaiyin Institute of Technology, Huai'an, P. R. China \\ E-Mail: shyhits@126.com
}

\begin{abstract}
In order to improve the efficiency and safety level of urban expressway, speed guidance control was utilized to optimize the traffic flow. Inner ring expressway of Shanghai $5 \mathrm{~km}$ long was modelled in VISSIM and conflict statistical analysis was carried out in SSAM based on the data of vehicles trajectory. The collaborative optimization method to intervene the crash risk using speed guidance control was given. Log-linear model was established according to the speed-related variables and the model was adopted as the objective function of quantify traffic safety. Then simulation was carried out integrating MATLAB, VISSIM and VB.NET under extended macroscopic dynamic traffic flow model. Speed guidance control system oriented quantified traffic safety for urban expressway using model predictive control was established. Then numerical simulation was launched, the results show that total traffic conflicts decrease $29.1 \%$, travel time of monitoring link reduces $45.3 \%$, and variance of travel time reduces $47.8 \%$. Under the speed guidance control, safety and efficiency of expressway both have been improved, which has important reference for delicacy traffic control and management. (Received, processed and accepted by the Chinese Representative Office.)
\end{abstract}

Key Words: Urban Expressway, Speed Guidance Control, Conflict Analysis, Log-Linear Model, Model Predictive Control

\section{INTRODUCTION}

Expressway system has become the urban mass rapid transit corridor and the main framework of urban road systems. Decreasing delay and improving throughput performance are great significance to improve the operation efficiency of expressway system. As the backbone of the city road network, urban expressway shares large proportion of the traffic. In Beijing, major urban expressway accounts for only $8 \%$ of the total length, but carries nearly $50 \%$ of the traffic flow; in Shanghai, only $5 \%$ bears more than $35 \%$ of the city traffic traveling $[1,2]$. Urban expressway plays a vital role in the urban road network which gradually shifted from the large-scale infrastructure construction to refinement traffic management. With the traffic demand rapid growing, much more congestion and traffic accidents, integrated active traffic management should be introduced in the background of coordination between road and vehicle. As an important part of the active traffic management, the speed guidance control has certain positive significance to improve the expressway capacity, reduce the accident risk and decrease even eliminates traffic congestion. Road safety assessment can be divided into direct and indirect methods. Traffic conflict technique is the representative method of the indirect traffic safety evaluation which has obvious advantages, relatively large number of conflict, short period and strong regularity. It was extremely widely applied in the field of road traffic safety. However, traffic conflict observed manually always was arbitrary, taking a lot of manpower and resources. Therefore surrogate safety assessment model (SSAM) which was developed by the United States Federal Highway Administration was used to analyse micro-simulation model output trajectory file. SSAM attempted to analyse the road security using simulation methods $[3,4]$. In recent years, with the development of Vehicles Infrastructure Integration system, taking active traffic management to 
expressway has become the research hotspots in this subject. The U.S. Department of Transportation Five Year ITS Strategic Research Plan clearly pointed out that dynamic speed harmonization would be one of the main means to optimize traffic flow in urban transportation networks. The Ministry of Science and Technology of China has put intelligent traffic management system as one of the key research areas in the national science and technology plan [5].

With the recent advances in active transportation and demand management, variable speed limits (VSL) have been identified as an active traffic management strategy for improving expressway mobility and safety. Several intelligent VSL strategies have been proposed and evaluated. The first known experiments with variable speed limit signs took place on a $30 \mathrm{~km}$ stretch of German motorway A8 between Munich and the border city of Salzburg, Austria. Mechanically variable message signs could display speeds of 60,80 and $100 \mathrm{~km} / \mathrm{h}$. Personnel monitored traffic using video technology, and manually controlled the signage. In an actual variable speed limit system, speed limits vary strategically and periodically in accordance with traffic volume and weather conditions to achieve better safety, efficiency, and environmental sustainability on urban expressway. Speed and speed variance of vehicles are important factors related to safety, especially during severe weather conditions [6]. According to the earlier variable speed limits studies, drivers generally choose the driving speed among the range, which is essentially a method of wide range speed control. Based on the earlier variable speed limits studies shortcoming, this paper proposes an integrated speed guidance method using model predictive control oriented quantified traffic safety for urban expressway and develops on-line simulation. Speed guidance control aim to provide a unique speed value for drivers. Vehicle trajectory data was analysed through SSAM based on the actual expressway model established in VISSIM. Detailed analysis on the conflict was processed to construct the log-linear model which was taken as the objective function of predictive control. Typical segment of Shanghai expressway was taken as the example, dynamic online simulation which integrated VISSIM, MATLAB and VB.NET was developed, then multi-step model predictive control was carried out oriented quantified traffic safety. The main impact of variable speed limits on traffic flow is deemed to reduce the mean speed around the critical densities and speed differences among vehicles. But their control strategies are passive and simple rule-based. Unlike traditional methods of variable speed limits, this paper provides a clear speed guidance value, driver only need to follow speed value explicitly.

\section{PROBLEM BACKGROUND}

Expressway system has become the urban mass rapid transit corridor and the framework of urban road systems. Ramp metering is the main method to control expressway traffic at present. Papageorgiou reviewed ramp metering algorithms researches comprehensively. The classical and widely practiced ramp metering method is ALINEA, which is a feedback control method. Ramp metering can control the flow into the expressway, but after entering the expressway the effect of using ramp metering alone to improve the traffic flow is not very well [7-10]. In recent years, taking active traffic management to expressway has become the development trend and one of the main methods is utilizing variable speed limits to control mainline traffic. In fact, there have been several VSL applications in Germany, Netherlands, UK and other counties in Europe [11-13]. Bertini evaluated the VSL strategy used at Autobahn 5 in Germany using the empirical data, the result showed that the effectiveness of VSL strategy in reducing congestion at bottlenecks was significant [14]. On the other hand, some VSL experiment had been carried out which concluded that VSL experiment had a significant effect in reducing speed variation and the number of shockwaves [15-19]. Representative researches of Hegyi have shown that 
the traffic shock wave can be better smoothed by VSL under MPC [20]. Despite a lot of progress, these studies did not reveal the quantitative effect of VSL towards the traffic safety. So based on the accident data, Lee established a model which was used for comparative analysis the risk changes before and after the VSL controls [21]. With the same model, Allaby analysed the road safety effects under different traffic demand by VSL controls [22]. According to different traffic speed level, Abdel-Aty founded two kinds of dynamic accident prediction models which used in the simulation analysis for different VSL control strategies [23]. The effects of VSL on traffic flow were studied using fundamental traffic model and shockwave theory [24, 25]. There are some limitations using single VSL control method, and then ramp metering was coordinated to achieve better control effect $[26,27]$. In addition, some new models were involved in VSL research [28-31]. Studies have shown that VSL has good control effect on greenhouse gas emissions [32, 33]. Driver obedience for the speed guidance value affected the effect directly. The effect might also lose even play a negative role when the traffic demand reached a certain level [34-37]. Models of these researches were established under accident data, so it requires a lot of high-quality traffic accident data. Meanwhile, control strategies are based on default rules, not the quantify traffic safety-oriented. VSL control method has essential uncertain characteristics about speed limits value for drivers; they generally choose the driving speed among a range. For example, if the value of VSL is $80 \mathrm{~km} / \mathrm{h}$, then vehicle speed not exceeding $80 \mathrm{~km} / \mathrm{h}$ is legitimate, may be $30 \mathrm{~km} / \mathrm{h}$, $50 \mathrm{~km} / \mathrm{h}$ or $60 \mathrm{~km} / \mathrm{h}$ which will cause the control not precisely enough. This paper focused on speed guidance control which provides the driver with a clear speed value which is the biggest difference compared with others research. On the other hand, Conflict statistical analysis was carried out in SSAM based on the data of vehicles trajectory. Finally, the collaborative optimization method to intervene the crash risk using speed guidance control was given. Log-linear model was established according to the speed-related variables and the model was adopted as the objective function of quantify traffic safety. Then MPC is adopted to compute the speed guidance values which orientate the safer level traffic flow.

\section{CONFLICTS LOG-LINEAR MODEL}

Road safety assessment can be divided into direct and indirect methods. Direct assessment method was based on accident statistics which was widely used in road traffic management. Though the method was simple to operate, it also had some disadvantages, such as relatively small of the road traffic accidents data, relatively long of the statistical period, great randomness of the accident and other problems. As one of the most important indirect methods, traffic conflict technique has the characteristics of relative large number conflicts, short statistical cycle and strong regularity. It was widely used in the field of road traffic safety research. But traffic conflict survey requires a lot of manpower resources and it was always the arbitrary result to some extent. Therefore, the U.S. Federal Highway Administration has developed a security analysis model SSAM to analyze the trace files of the simulation data, which can be reached the result of safety evaluation through the traffic simulation data.

Dynamic risk of accident was quantified analysed during operation based on real-time traffic data. The higher accident risk, the greater the likelihood of an accident, then measures should be taken to control the seemingly safe traffic flow. The dynamic accident risk model was used to analyse the possibility of potential accident and control the traffic flow based on model predictive control. SSAM was adopted to analyse the conflicts by the vehicle trajectory provided by VISSIM simulation result. Several factors were considered to establish the model, space velocity difference, time velocity difference, road geometric 
characteristics and traffic volume. Based on this classification, the time speed difference (SVT), space velocity difference (SVD), road geometry (RD), the road traffic service (TS) were treated as the independent variables; serious conflict quantity (DAR) was treated as the dependent variable, which was the dynamic quantization value of traffic accident risk.

The log-linear model was listed as eq. (1):

$$
\ln (D A R)=\alpha+L_{S V T(i)}+L_{S V D(j)}+L_{T S(m)}+L_{R G(n)}
$$

where $\alpha$ is the total average effect, $L_{S V T(i)}$ is time speed difference classification level index, $L_{S V D(j)}$ is space velocity difference classification level index, $L_{T S(m)}$ is the road traffic services classification level index, $L_{R G(n)}$ is the road geometry classification level index.

\section{DYNAMIC TRAFFIC FLOW MODEL}

Control-oriented macro dynamic traffic flow model describes the relationship among traffic flow over space and time even traffic control variables. LW model was proposed by British scholar Lighthill and Whitham in 1955. Against the defects, Payne proposed dynamic relationship between speed and density. The model was further extended considering off-ramp, on-ramp and lane change factors by Papageorgiou. Scholars between domestic and foreign have also proposed various models which are mostly around dynamic relationship between speed and density. Model proposed by Payne and Papageorgiou are widely used in practice. Second order dynamic METANET model is used to describe the traffic flow. To make the calculated optimal speed guidance value more realistic and accurate, dynamic traffic flow model are described as eqs. (2) and (3):

$$
\begin{gathered}
\rho_{i}(k+1)=\rho_{i}(k)+\frac{T}{L_{i} \lambda_{i}}\left(\rho_{i-1}(k) v_{i-1}(k)-\rho_{i}(k) v_{i}(k)+r_{i}(k)-s_{i}(k)\right) \\
v_{i}(k+1)=v_{i}(k)+\frac{T}{\tau}\left(u_{i}(k)-v_{i}(k)\right)+\frac{T}{L_{i}} v_{i}(k)\left(v_{i-1}(k)-v_{i}(k)\right)-\frac{1}{\tau}\left(\frac{v T}{L_{i}} \frac{\rho_{i+1}(k)-\rho_{i}(k)}{\rho_{i}(k)+\kappa}\right)
\end{gathered}
$$

where $i$ is road segment index, $k$ is time interval index, $T$ is the time step used for data collection, $L_{i}$ is length of road segment, $\tau, v, \kappa$ are the model parameters, $\tau$ is a time constant, $v$ is the anticipation constant and $\kappa$ is model parameter, which are equal at a segment. $\rho_{i}(k)$ is the traffic density of road segment $i$ at time index $k, v_{i}(k)$ is the mean speed of road segment $i$ at time index $k, r_{i}(k)$ is the metering flow rate of road segment $i$ at time index $k, s_{i}(k)$ is the total off-ramp flow rate of road segment $i$ at time index $k, u_{i}(k)$ is the desired control speed of road segment $i$ at time index $k$ and $\lambda_{i}$ is the number of lanes of road segments.

The paper determines the origins flow and upstream speed parameters by the ramp length and queue of upstream cell segment. The models are listed as eqs. (4) to (6):

$$
\begin{gathered}
w_{0}(k+1)=w_{0}(k)+T\left(d_{0}(k)-q_{0}(k)\right) \\
q_{0}(k)=\min \left[d_{0}(k)+w_{0}(k) / T, Q_{0} \frac{\rho_{\max }-\rho_{\mu, 1}(k)}{\rho_{\max }-\rho_{\text {crit }, \mu}}\right] \\
v=\min \left[(1+\alpha) v_{\text {crit }, m}(k), v_{\text {free }} \exp \left[-\frac{1}{\alpha m}\left(\frac{\rho_{\mu, 1}(k)}{\rho_{\text {crit }, u}}\right)^{\alpha m}\right]\right]
\end{gathered}
$$

where $Q_{0}$ is the onramp flow capacity, $\rho_{\max }$ is the maximum density of onramp, $\rho_{\text {crit, } \mu}$ is the critical density of onramp at which the traffic flow becomes unstable, $\rho_{\mu, 1}(k)$ is the density of mainline which segment the onramp linked, $v_{c r i t, m}$ is the critical speed of mainline at which the traffic flow becomes unstable, $v_{\text {free }}$ is the speed of freely traffic flow, $w_{0}(k)$ is the maximum number of vehicles stored in onramp and $d_{0}(k)$ is the onramp demand flow. 
Considering traffic safety, speed change should be smoothing both temporally and spatially. So the control speed variation should be less than $10 \mathrm{~km} / \mathrm{h}$ over time or distance interval, the following constraints are adopted in eq. (7):

$$
\bar{V}_{\min } \leq u_{i}(k) \leq \bar{V}_{\max }
$$

where $\bar{V}_{\min }$ and $\bar{V}_{\max }$ are the minimum and maximum control speed for speed guidance control respectively; $\bar{V}_{\min }$ taken as $20 \mathrm{~km} / \mathrm{h}$ and $\bar{V}_{\max }$ taken as $80 \mathrm{~km} / \mathrm{h}$.

\section{SIMULATION ANALYSIS AND RESULTS}

In the speed guidance control online simulation system, VISSIM software is used to simulate the real world traffic. The macro dynamic traffic flow model is established in MATLAB. Data and control strategies were exchange through the API interface among VB.NET, VISSIM and MATLAB.

According to the functional orientation, online simulation system was divided into four modules: simulation module, strategy module, interface module and database module. The structures of the simulation system as well as the relationship among the modules were shown in Fig. 1.

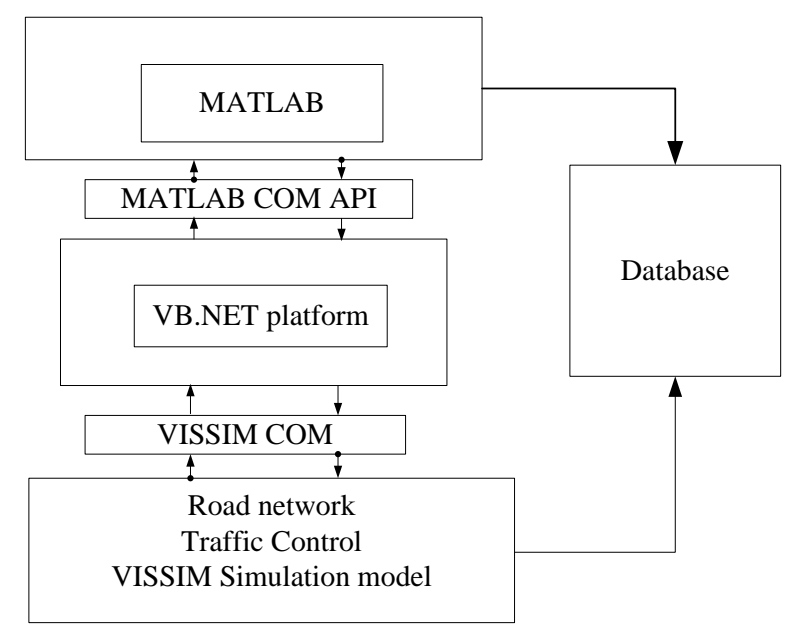

Figure 1: Framework of online simulation.

Inner ring expressway in Shanghai was selected as the research object shown in Fig. 2. It was $5 \mathrm{~km}$ long and divided into eight sections with traffic parameters detectors located downstream and upstream. Simulation model was established, calibration and verification.

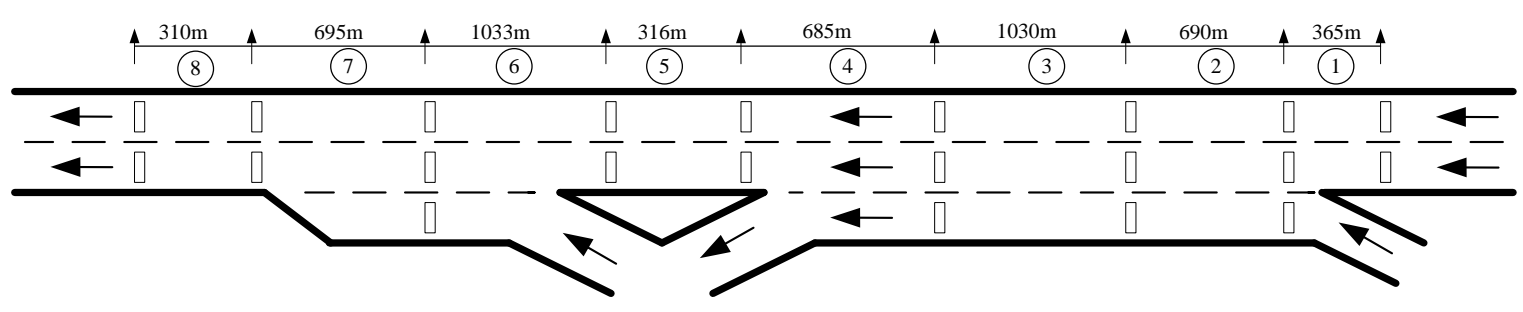

Figure 2: Abstract figure of urban expressway.

Vehicle trajectory data provided by VISSIM simulation result was analysed through SSAM. Then SPSS was involved to establish the log-linear model. Distribution of SVT and SVD values were showed in Fig. 3. SVT was divided into three categories, first 
category is the detected speeds almost no change in time, the second is detected speeds have a smaller fluctuation in time, and the third category is that there are relatively large fluctuations in detected speeds. According to descending order and the $60 \%, 30 \%, 10 \%$ percentage value, three classification intervals were built:

$\left|v_{t+1}-v_{t}\right|<1 \mathrm{~km} / \mathrm{h}, 1 \mathrm{~km} / \mathrm{h} \leq\left|v_{t+1}-v_{t}\right|<3 \mathrm{~km} / \mathrm{h}$ and $\left|v_{t+1}-v_{t}\right| \geq 3 \mathrm{~km} / \mathrm{h}$.

Most of the space velocity differences were within the interval $[-10,10]$. On the other hand, considering the control step of speed guidance, SVD values were divided into three categories, less than $-10 \mathrm{~km} / \mathrm{h}$, greater than $10 \mathrm{~km} / \mathrm{h}$ and within the range $-10 \mathrm{~km} / \mathrm{h}$ to $10 \mathrm{~km} / \mathrm{h}$.
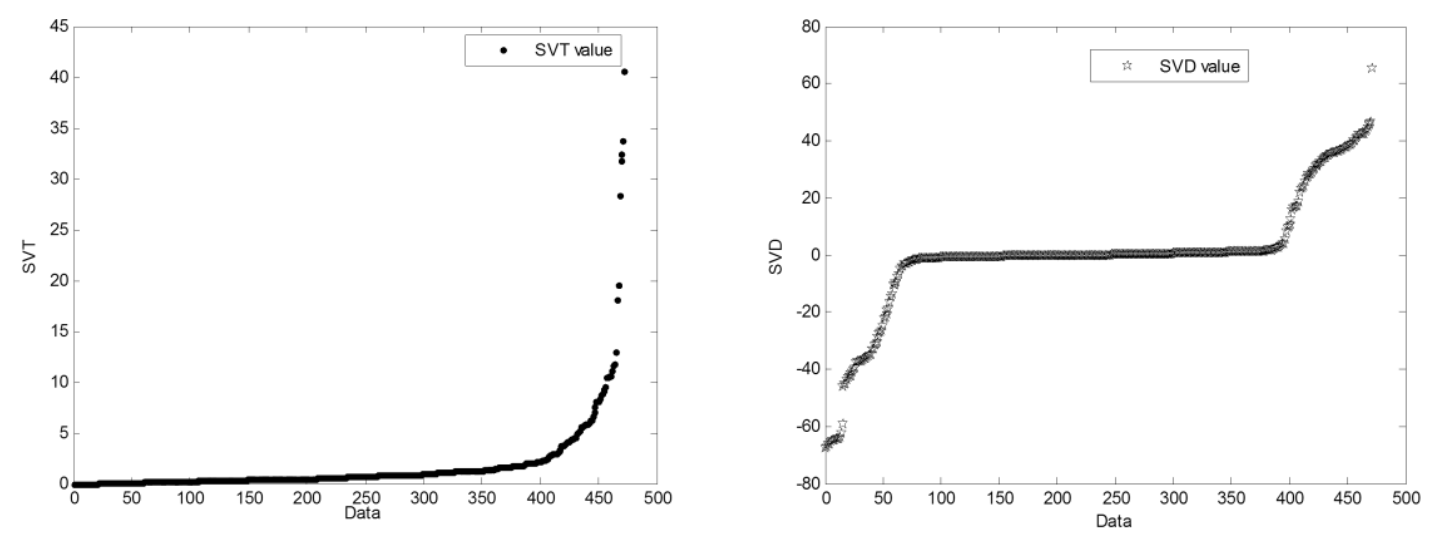

Figure 3: Distribution of SVT and SVD.

Average value of road traffic was $20 \mathrm{veh} / \mathrm{min} /$ lane corresponding to $1200 \mathrm{veh} / \mathrm{h} / \mathrm{lane}$. While road capacity was $2000 \mathrm{veh} / \mathrm{h} / \mathrm{lane}, 1200 \mathrm{veh} / \mathrm{h} / \mathrm{lane}$ was selected as the threshold value to distinguish different traffic classification.

The expressway was divided into eight sections; section 1 was the initial segment which also contained entrance ramp. Section 4 included exit ramp, section 6 contained entrance ramp, and section 7 contained transition part. Therefore road geometry was divided into two categories, one classification included section 2, 3, 5, 8 and the other included section $1,4,6,7$.

Table I: Parameter estimates.

\begin{tabular}{|c|c|c|c|c|}
\hline Parameter & Estimate & Std. Error & Lower Bound & Upper Bound \\
\hline Constant & 7.625 & 0.010 & 7.605 & 7.645 \\
\hline$[\mathrm{SVT}=1]$ & 0.791 & 0.010 & 0.773 & 0.810 \\
\hline$[\mathrm{SVT}=2]$ & 0.872 & 0.009 & 0.853 & 0.891 \\
\hline$[\mathrm{SVT}=3]$ & 0 & 0 & 0 & 0 \\
\hline$[\mathrm{SVD}=1]$ & 0.058 & 0.007 & 0.043 & 0.072 \\
\hline$[\mathrm{SVD}=2]$ & -1.028 & 0.010 & -1.048 & -1.008 \\
\hline$[\mathrm{SVD}=3]$ & 0 & 0 & 0 & 0 \\
\hline$[\mathrm{TS}=1]$ & 0.711 & 0.007 & 0.697 & 0.725 \\
\hline$[\mathrm{TS}=2]$ & 0 & 0 & 0 & 0 \\
\hline$[\mathrm{RG}=1]$ & -2.981 & 0.016 & -3.012 & -2.950 \\
\hline$[\mathrm{RG}=2]$ & 0 & 0 & 0 & 0 \\
\hline
\end{tabular}

Log-linear model was established, time speed difference and space speed difference both were three classification levels. Road geometry and traffic volume were two classification levels. There were total 36 kinds of combinations of individual combinations; modelling and analysis were processing in SPSS by frequency statistics. It was supposed that conflict was random and Poisson distribution. Log-linear model was 
established based on the number of the conflicts and main effects analysis was carried out simultaneously. The value of the main effects reflect its contribution to the expected frequencies, which only demonstrate the different of row or column variable and do not reflect the relationship between the other variables. Results of model parameter estimation were shown in Table I.

The value of Sig was close to zero indicating that selected variables were affected the conflicts significantly. Some classification model parameter was zero, because the main effect variable estimated under the constraint that one variable value was zero. The constant parameter estimated value of the model was 7.625. Estimated values of time speed difference were $0.791,0.872$ and 0 respectively, which indicated that $1 \mathrm{~km} / \mathrm{h} \leq\left|v_{t+1}-v_{t}\right|<3 \mathrm{~km} / \mathrm{h}$ classification level was the most serious impact on the conflicts. Time speed difference should avoid frequent fluctuations in small amplitude. Estimated values of SVD were $0.058,-1.028$ and 0 respectively, which indicated that SVD less than $-10 \mathrm{~km} / \mathrm{h}$ classification level was the most serious impact on the conflicts. Traffic accident was easy to occur when speed drop dramatically. Two classifications level parameter estimation of road traffic service were 0.711 and 0 respectively. When the road traffic was relatively low, less than $1200 \mathrm{veh} / \mathrm{h} /$ lane, more conflicts occurred. Estimated values of RG were -2.981 and 0 respectively indicating that more conflicts might occur near the entrances, exits and lane change sections. Impacts of SVT, SVD, TS and RD for the conflicts were revealed after the founding of log-linear model which also used for model predictive control as the evaluation of safety.

To solve the problem of speed guidance control we apply the model predictive control scheme in a rolling framework shown in Fig. 4. In this control scheme a discrete-time model was used to predict the future behaviour of the process. The goal of the controller is to find the control signals that result in an optimal traffic behaviour. To express performance an objective function was defined and the speed guidance control signals that minimize this function were found via optimization. The objective function was shown in eq. (8):

$$
F=T \sum_{k=1}^{N_{2}-1}\left\{\sum_{i=1}^{N_{3}} \rho_{i}(k) L_{i} \lambda_{i}+w_{i}(k)\right\}+\beta \sum_{k=1}^{N_{1}-1} \sum_{i=1}^{N_{3}}\left(D A R_{i}(k)-D A R_{i}(k-1)\right)^{2}
$$

where $N_{1}$ is the control step size; $N_{2}$ is the prediction step size; $\beta$ is the weight coefficient and $N_{3}$ is the number of road sections.

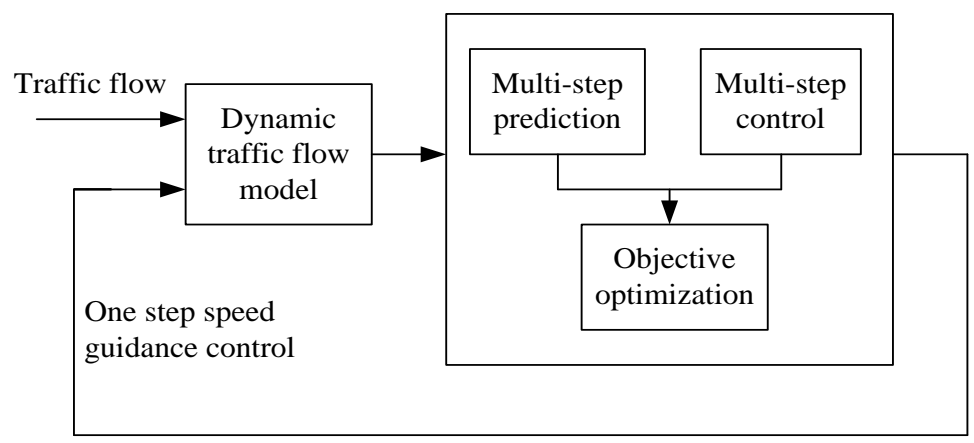

Figure 4: Process of speed guidance control.

The control was applied in a rolling horizon scheme. At each time instant a new optimization was performed over the prediction horizon, and only the first value of the resulting control signal was applied to the process. The next time instant the same procedure is repeated. To reduce complexity and improve stability a control horizon was introduced, and after the control horizon has been passed the control signal is taken to be 
constant. The advantage of this rolling horizon approach was that resulted in an on-line adaptive control scheme which allows us to take changes in the system parameters into account by regularly updating the model of the system.

The expressway was divided into eight sections; each part has speed guidance control respectively. The cycle of speed guidance control was $1 \mathrm{~min}$, so the traffic flow model iteration number was 6 . Then control step size was 42 and prediction step size was 60 . Road traffic demand is shown in Fig. 5. Results of speed guidance value are shown in Fig. 6 and comparative analysis is shown in Table II.

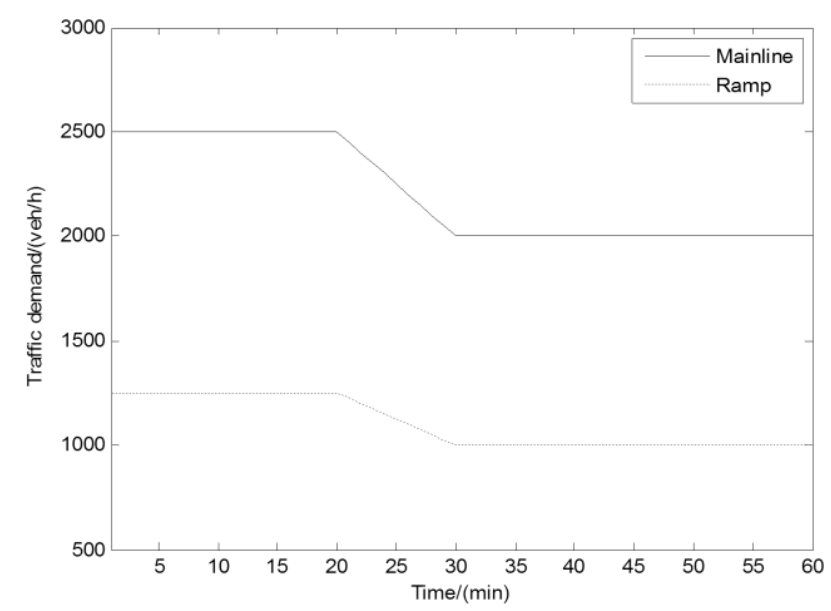

Figure 5: Traffic demand.

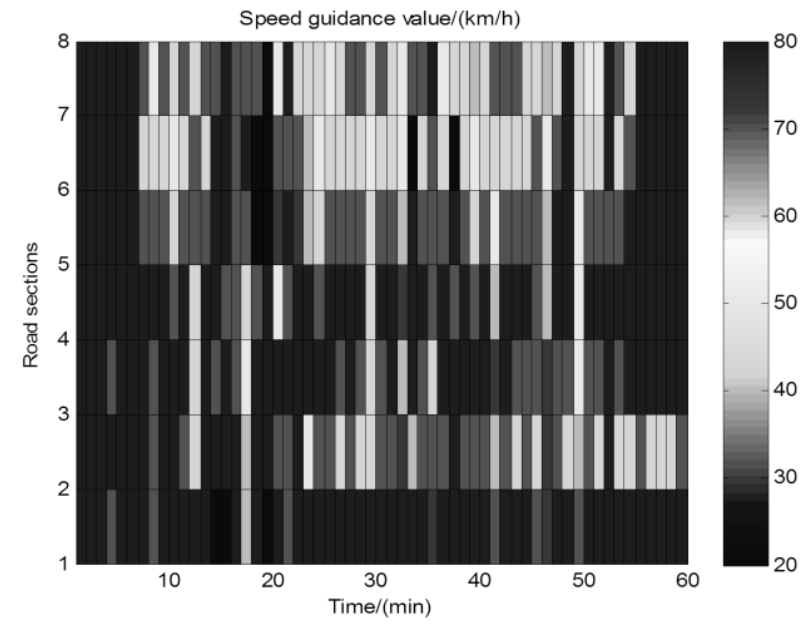

Figure 6: Speed guidance value.

Table II: Simulation results.

\begin{tabular}{|l|c|c|c|}
\hline \multicolumn{1}{|c|}{ Results } & No control & Under control & Effect \\
\hline Conflicts & 257038 & 182171 & $-29.1 \%$ \\
\hline Average travel time & 249.3 & 136.3 & $-45.3 \%$ \\
\hline Standard deviation of travel time & 72.2 & 37.7 & $-47.8 \%$ \\
\hline
\end{tabular}

Under the speed guidance control oriented quantified safety, the simulation results show that the number of conflicts decreased by $29.1 \%$, average travel time reduced by $45.3 \%$ and standard deviation of travel time decreased by $47.8 \%$. The results indicated that both safety and efficiency of the simulation sections have been improved. Fluctuations of the travel time decreased revealed that its reliability has also been enhanced. 


\section{CONCLUSIONS}

This study aims to investigate the principle of speed guidance control and the effect on traffic flow using model predictive control. Speed changed relative smoothly when the urban expressway controlled under speed guidance. It is necessary to evaluate the effect on traffic safety. Furthermore, speed guidance control should follow the principle of quantified traffic safety. Vehicle trajectory was analysed through SSAM based on the actual expressway model established in VISSIM. Detailed analysis on the conflict was handled. The log-linear model was established considering the factors affected the velocity. Model predictive control was involved to optimize the speed value which was safety-oriented. The crash risk generally forms by dynamic random traffic flow, static traffic facilities and unreasonable traffic control strategy which is the potential factor induced the crash. Traditional research focus on the realtime control after the crash had happened which are lack of predictability, delaying in response and not considering the countermeasures for real-time crash risk. Based on the demand of real-time control, speed guidance is introduced to establish the dynamic control model in order to grasp the possibility and severity of the crash risk. Then collaborative optimization method to intervene the crash risk using speed guidance control is built. Feedback between design framework program and real-time program is processing utilized active traffic management mechanism. Single-point value of speed guidance control is explored and optimization strategy about multi-point values is also established considering physical variables and speed derived variables. Software integrated environment is building to identify crash risk and apply dynamic speed guidance control strategy. Control strategy of speed guidance by crash risk intervening for urban expressway is created. The results will reveal the discipline of expressway crash risk and provide theoretical support and technical basis in reducing risk, preventing block and controlling congestion. Under the speed guidance control, safety and efficiency of expressway both have been improved which has important reference for delicacy traffic control and management.

However, there are remains rooms that needed future endeavours. First of all, more safety factors should be taken into account in the log-linear model to form a more complete quantitative safety objective function. The length of the road section was decided considering the reference researches which pay close attention to the physical properties of the expressway. Dividing the length of the road into different sections will inevitably lead to different results, and then the effect of speed guidance control was diversification. Optimal control model based on length of the road needs to establish in the future. Second, obedience of the driver to the speed guidance value directly affects the effect; the effect might also lose even play a negative role in some cases, so we should consider the impact of the rate that drivers obey the information in reality. Though efficiency and security had been taking into account in the research, environmental benefits were ignored. As a very important direction of future research, speed guidance control based on ecological driving is required to establish the models and strategies. At last, applying the method presented in the paper to the actual speed management and control on the real expressway system, and this work is underway.

\section{ACKNOWLEDGEMENTS}

The authors would like to thank the Department of Traffic Engineering, Tongji University and Sino-German Transportation Research Center for their support in the research work. This research was supported by the science and technology projects of MOHURD (No. 2013-K5$3)$. 


\section{REFERENCES}

[1] Zhao, N.; Yu, L.; Zhao, H.; Guo, J.; Wen, H. (2009). Analysis of traffic flow characteristics on ring road expressways in Beijing using floating car data and remote traffic microwave sensor data, Transportation Research Record, Vol. 2124, No. 1, 178-185, doi:10.3141/2124-17

[2] Lu, X.-Y.; Varaiya, P.; Horowitz, R.; Su, D.; Shladover, S. E. (2011). Novel freeway traffic control with variable speed limit and coordinated ramp metering, Transportation Research Record, Vol. 2229, No. 1, 55-65, doi:10.3141/2229-07

[3] Lee, C.; Abdel-Aty, M. (2008). Testing effects of warning messages and variable speed limits on driver behavior using driving simulator, Transportation Research Record, Vol. 2069, No. 1, 55 64, doi:10.3141/2069-08

[4] Abdel-Aty, M.; Cunningham, R. J.; Gayah, V. V.; Hsia, L. (2008). Dynamic variable speed limit strategies for real-time crash risk reduction on freeways, Transportation Research Record, Vol. 2078, No. 1, 108-116, doi:10.3141/2078-15

[5] Wang, Y. Z.; Zhang, Y.; Hu, J. M.; Li, L. (2012), Using variable speed limits to eliminate wide moving jams: a study based on three-phase traffic theory, International Journal of Modern Physics C, Vol. 23, No. 9, 16 pages, doi:10.1142/S012918311250060X

[6] Papageorgiou, M.; Kosmatopoulos, E.; Papamichail, I. (2008). Effects of variable speed limits on motorway traffic flow, Transportation Research Record, Vol. 2047, 37-48, doi:10.3141/2047-05

[7] Papageorgiou, M.; Papamichail, I. (2008). Overview of traffic signal operation policies for ramp metering, Transportation Research Record, Vol. 2047, No. 1, 28-36, doi:10.3141/2047-04

[8] Papageorgiou, M.; Kotsialos, A. (2002). Freeway ramp metering: an overview, IEEE Transactions on Intelligent Transportation Systems, Vol. 3, No. 4, 271-281, doi:10.1109/ TITS.2002.806803

[9] Papageorgiou, M.; Hadj, S. H.; Blosseville J. M. (1991). Alinea: a local feedback control law for on ramp metering, Transportation Research Record, Vol. 1320, No. 1, 58-67

[10] Carlson, R. C.; Papamichail, I.; Papageorgiou, M.; Messmer, A. (2010). Optimal motorway traffic flow control involving variable speed limits and ramp metering, Transportation Science, Vol. 44, No. 2, 238-253, doi: $10.1287 /$ trsc. 1090.0314

[11] Song, Q. H.; Ai, X.; Tang, W. X. (2011). Prediction of simultaneous dynamic stability limit of time-variable parameters system in thin-walled workpiece high-speed milling processes, International Journal of Advanced Manufacturing Technology, vol. 55, No. 9-12, 883-889, doi:10.1007/s00170-010-3139-8

[12] Fudala, N. J.; Fontaine, M. D. (2010). Interaction between system design and operations of variable speed limit systems in work zones, Transportation Research Record, Vol. 2169, No. 1, 1-10, doi:10.3141/2169-01

[13] Buddemeyer, J.; Young, R. K.; Dorsey-Spitz, B. (2010). Rural variable speed limit system for Southeast Wyoming, Transportation Research Record, Vol. 2189, No. 1, 37-44, doi:10.3141/ 2189-05

[14] Bertini, R. L.; Boice, S.,; Bogenberger, K. (2006). Dynamics of variable speed limit system surrounding bottleneck on German Autobahn, Transportation Research Record, Vol. 1978, No. 1, 149-159, doi:10.3141/1978-20

[15] Hadiuzzaman, M.; Qiu, T. Z.; Lu, X. Y. (2012). Variable speed limit control design for relieving congestion caused by active bottlenecks, Journal of Transportation Engineering, Vol. 139, No. 4, 358-370, doi:10.1061/(ASCE)TE.1943-5436.0000507

[16] Jo, Y.; Kim, Y.; Jung, I. (2012). Variable speed limit to improve safety near traffic congestion on urban freeways, International Journal of Fuzzy Systems, Vol. 14, No. 2, 278-288

[17] Chiou, Y.-C.; Huang, Y.-F.; Lin, P.-C. (2012). Optimal variable speed-limit control under abnormal traffic conditions, Journal of the Chinese Institute of Engineers, Vol. 35, No. 3, 299308, doi:10.1080/02533839.2012.655472

[18] Heydecker, B. G.; Addison, J. D. (2011). Analysis and modelling of traffic flow under variable speed limits, Transportation Research Part C: Emerging Technologies, Vol. 19, No. 2, 206-217, doi:10.1016/j.trc.2010.05.008

[19] Chang, G.-L.; Park, S. Y.; Paracha, J. (2011). Intelligent transportation system field demonstration - Integration of variable speed limit control and travel time estimation for a 
recurrently congested highway, Transportation Research Record, Vol. 2243, No. 1, 55-66, doi:10.3141/2243-07

[20] Hegyi, A.; De Schutter, B.; Hellendoorn, H. (2005). Model predictive control for optimal coordination of ramp metering and variable speed limits, Transportation Research Part C: Emerging Technologies, Vol. 13, No. 3, 185-209, doi:10.1016/j.trc.2004.08.001

[21] Lee, C.; Hellinga, B.; Saccomanno, F. (2006). Evaluation of variable speed limits to improve traffic safety, Transportation Research Part C: Emerging Technologies, Vol. 14, No. 3, 213-228, doi:10.1016/j.trc.2006.06.002

[22] Allaby, P.; Hellinga, B.; Bullock, M. (2007). Variable speed limits: safety and operational impacts of a candidate control strategy for freeway applications, IEEE Transactions on Intelligent Transportation Systems, Vol. 8, No. 4, 671-680, doi:10.1109/TITS.2007.908562

[23] Abdel-Aty, M; Dilmore, J.; Dhindsa, A. (2006). Evaluation of variable speed limits for real-time freeway safety improvement, Accident Analysis and Prevention, Vol. 38, No. 2, 335-345, doi:10.1016/j.aap.2005.10.010

[24] Cho, H.; Kim, Y. C. (2012). Analysis of traffic flow with variable speed limit on highways, KSCE Journal of Civil Engineering, Vol. 16, No. 6, 1048-1056, doi:10.1007/s12205-012-1395-x

[25] Kononov, J.; Durso, C.; Reeves, D.; Allery, B. K. (2012). Relationship between traffic density, speed, and safety and its implications for setting variable speed limits on freeways, Transportation Research Record, Vol. 2280, No. 1, 1-9, doi:10.3141/2280-01

[26] Lu, X.-Y.; Varaiya, P.; Horowitz, R.; Su, D. (2010). A new approach for combined freeway variable speed limits and coordinated ramp metering, $13^{\text {th }}$ International IEEE Conference on Intelligent Transportation Systems, 491-497, doi:10.1109/ITSC.2010.5625107

[27] Frejo, J. R. D.; Camacho, E. F. (2012). Global versus local MPC algorithms in freeway traffic control with ramp metering and variable speed limits, IEEE Transactions on Intelligent Transportation Systems, Vol. 13, No. 4, 1556-1565, doi:10.1109/TITS.2012.2195493

[28] Zhang, J. J.; Pang, M. B.; Ren, S. S. (2012). Characteristic analysis of traffic flow in variable speed limit section of freeway based on cellular automaton model, Acta Physica Sinica, Vol. 61, No. 24, 340-347, doi:10.7498/aps.61.244503

[29] Carlson, R. C.; Papamichail, I.; Papageorgiou, M. (2012). Local feedback-based mainstream traffic flow control on motorways using variable speed limits, IEEE Transactions on Intelligent Transportation Systems, Vol. 12, No. 4, 1261-1276, doi:10.1109/TITS.2011.2156792

[30] Wang, Y.; Ioannou, P. A. (2011). New model for variable speed limits, Transportation Research Record, Vol. 2249, No. 1, 38-43, doi:10.3141/2249-06

[31] Hadiuzzaman, M.; Qiu, T. Z. (2013). Cell transmission model based variable speed limit control for freeways, Canadian Journal of Civil Engineering, Vol. 40, No. 1, 46-56, doi:10.1139/cjce2012-0101

[32] Liu, B. J.; Ghosal, D.; Chuah, C. N.; Zhang, H. M. (2012). Reducing greenhouse effects via fuel consumption-aware variable speed limit, IEEE Transactions on Vehicular Technology, Vol. 61, No. 1, 111-122, doi:10.1109/TVT.2011.2170595

[33] Bel, G.; Rosell, J. (2013). Effects of the $80 \mathrm{~km} / \mathrm{h}$ and variable speed limits on air pollution in the metropolitan area of Barcelona, Transportation Research Part D: Transport and Environment, Vol. 23, 90-97, doi:10.1016/j.trd.2013.04.005

[34] Long, S.; Gentry, L.; Bham, G. H. (2012). Driver perceptions and sources of user dissatisfaction in the implementation of variable speed limit systems, Transport Policy, Vol. 23, 1-7, doi:10.1016/j.tranpol.2012.05.007

[35] Hassan, H. M.; Abdel-Aty, M.; Choi, K.; Algadhi, S. (2012). Driver behavior and preferences for changeable message signs and variable speed limits in reduced visibility conditions, Journal of Intelligent Transportation Systems, Vol. 16, No. 3, 132-146, doi:10.1080/15472450.2012.691842

[36] Hellinga, B.; Mandelzys, M. (2011). Impact of driver compliance on the safety and operational impacts of freeway variable speed limit systems, Journal of Transportation Engineering, Vol. 137, No. 4, 260-268, doi:10.1061/(ASCE)TE.1943-5436.0000214

[37] Chen, D. S.; Zhang, Y.; Xia, L. G. (2013). Sensitivity simulation analysis of urban expressway under speed guidance control, Journal of Theoretical and Applied Information Technology, Vol. 48, No. $1,288-292$ 